\title{
FLIP is frequently expressed in endometrial carcinoma and has a role in resistance to TRAIL-induced apoptosis
}

\author{
Xavier Dolcet ${ }^{1}$, David Llobet ${ }^{1}$, Judit Pallares ${ }^{1}$, Montserrat Rue ${ }^{1}$, Joan X Comella ${ }^{2}$ and \\ Xavier Matias-Guiu ${ }^{1}$ \\ ${ }^{1}$ Department of Pathology and Molecular Genetics, Hospital Universitari Arnau de Vilanova, University of \\ Lleida, Spain and ${ }^{2}$ Cell Signalling and Apoptosis group, Laboratori de Recerca Biomedica, Dep of Ciencies \\ Mèdiques Bàsiques, Hospital Universitari Arnau de Vilanova, University of Lleida, Spain
}

\begin{abstract}
The FLICE-inhibitory protein (FLIP) plays a key role in the regulation of apoptosis triggered by death ligands. Tumor necrosis factor-related apoptosis-inducing ligand (TRAIL) has been shown to induce apoptosis in some types of tumor but not in others. To assess the possible role of FLIP in apoptosis resistance in endometrial carcinoma, we performed an immunohistochemical study on a tissue microarray composed of 95 endometrial carcinomas. We found positive signals in $43 \%$ of the cases, as well as a significant difference in FLIP expression between stage I and II tumors. Moreover, we observed that endometrial carcinoma cell lines Ishikawa and KLE did not undergo apoptosis after TRAIL treatment. Cotreatment of these cells with the inhibitor of transcription actinomycin D resulted in a dramatic decrease in cell viability and induced activation of caspase-8. These events coincided with downregulation of FLIP mRNA and protein. Inhibitors of caspase-8 or overexpression of FLIP completely blocked apoptosis induced by actinomycin D plus TRAIL cotreatment. More importantly, downregulation of endogenous FLIP expression by specific siRNAs sensitized endometrial carcinoma cells to TRAIL-induced apoptosis in the absence of actinomycin D. Taken together, our results suggest for the first time a critical role for FLIP in the regulation apoptosis triggered by TRAIL in endometrial carcinoma cells.
\end{abstract}

Laboratory Investigation (2005) 85, 885-894. doi:10.1038/labinvest.3700286; Published online 2 May 2005

Keywords: endometrial carcinoma; FLIP; TRAIL; tissue microarray, molecular pathology; apoptosis

Apoptosis is an important process that regulates homeostasis in multicellular tissues. Alterations in proteins that regulate apoptosis may result in impaired cell death and the development and progression of cancer. ${ }^{1}$ The tumor necrosis factorrelated apoptosis-inducing ligand (TRAIL) belongs to the proapoptotic cytokines of the tumor necrosis factor (TNF) superfamily. Several lines of evidence have shown that TRAIL induces apoptosis in many types of cancer with limited cytotoxicity on normal cells $^{2,3}$ indicating that it may become a promising anticancer agent. ${ }^{4}$ TRAIL can bind four different receptors DR4/TRAIL-R1, ${ }^{5,6}$ DR5/TRAIL-R2, ${ }^{7,8}$ DcR1/TRAIL-R3, ${ }^{9}$ and DcR2/TRAIL-R4. ${ }^{10}$ DR4 and DR5 are functional receptors that transduce

Correspondence: Professor X Matias-Guiu, MD, Department of Pathology and Molecular Genetics,Hospital Universitari Arnau de Vilanova, Av Alcalde Rovira Roure 80, 25198 Lleida, Spain.

E-mail: xmatias@arnau.scs.es

Received 28 January 2005; revised 22 March 2005; accepted 24 March 2005; published online 2 May 2005 apoptosis after ligation with TRAIL. DcR1 and DcR2, known as decoy receptors, lack the intracellular domains required to induce apoptosis. Recently, it has been shown that osteoprotegerin (OPG) can also bind TRAIL with less affinity. ${ }^{11}$

Engagement of receptors DR4 or Dr5 results in the formation of a death-inducing signalling complex (DISC). The intracellular death domain (DD) of these receptors recruits Fas associated DD-containing protein (FADD), which in turn binds procaspase-8. After recruitment to the DISC, procaspase-8 is activated by autoproteolytic cleavage resulting in the initiation of apoptotic signalling. ${ }^{12-14}$ Active caspase-8 activates the executioner caspase-3, which in turn cleaves the cellular substrates.

The FLICE-inhibitory protein (FLIP) was initially described as a homologue of viral v-FLIP, which is a key regulator of death-receptor signalling. ${ }^{15,16}$ Two isoforms of FLIP are generated by alternative splicing: the long form, FLIP-L; and the short form, FLIP-S. ${ }^{16}$ Both proteins share a high degree of homology with caspase-8, and contain two Death 
Effector Domains (DED). FLIP-L also contains a caspase-like domain but lacks proteolytic activity. Therefore, high levels of expression of FLIP result in inhibition of caspase-8 activation and apoptosis triggered by death receptors. Recent evidence indicates that FLIP is constitutively or highly expressed in some tumors such as prostate cancer, ${ }^{17}$ Hodgkin's lymphoma, ${ }^{18}$ gastric cancer, ${ }^{19}$ bladder carcinoma ${ }^{20}$ and malignant mesothelial cell lines. ${ }^{21}$

Although TRAIL induces apoptosis in a wide variety of neoplastic cells, certain tumors are resistant to TRAIL killing. The potential use of TRAIL in cancer therapy increases interest in the understanding of the molecular mechanisms by which tumor cells are resistant to TRAIL.

In this article, we provide evidence for the first time that suggests that FLIP may be important in apoptosis resistance in endometrial carcinoma. First, we show that FLIP is frequently expressed in endometrial carcinoma and correlates with stage. Moreover, we show that Ishikawa and KLE cell lines are resistant to TRAIL-induced apoptosis. Inhibition of transcription by actinomycin D results in cell viability decrease and induction of apoptosis. The sensitization to TRAIL by actinomycin D correlated with a significant reduction on FLIP expression and forced expression of FLIP overcame TRAIL plus actinomycin D-induced apoptosis. Moreover, downregulation of endogenous FLIP by siRNA was sufficient to sensitize endometrial carcinoma cells to TRAIL-induced apoptosis.

\section{Materials and methods}

\section{Tissue Microarray Construction and Immunohistochemistry}

A tissue microarray (TMA) was constructed from paraffin-embedded blocks of 95 endometrial carcinomas (EC), obtained from the Department of Pathology of Hospital Santa Creu i Sant Pau, Barcelona, Spain. The specimens were collected between 1996 and 2001. The tumors were classified by following the World Health Organization (WHO) criteria. They were surgically staged and graded according to the International Federation of Gynecology and Obstetrics (FIGO) staging and grading systems. The specimens included: 25 endometrioid carcinomas (EEC) grade I, 34 EEC grade II, 19 EEC grade III, 10 serous carcinomas (SC), 4 clear cell carcinomas (CC) and the epithelial components of three malignant mixed Müllerian tumors (MMMT). Totally about 65 cases were stage I, four were stage II, 14 were stage III, and one was stage IV. The study was approved by the local Ethical Committee.

A tissue array device (Beecher Instruments, MD, USA) was used. All ECs were histologically reviewed and representative tumor areas were marked in the corresponding paraffin blocks. Two selected cylinders $(0.6 \mathrm{~mm}$ in largest diameter) from two different tumor areas were included for each case.
Normal control tissues from the same specimens were also included. Thus, two different TMAs blocks were constructed; each of them contained 186 cylinders.

TMA blocks were sectioned at a thickness of $3 \mu \mathrm{m}$, dried for $16 \mathrm{~h}$ at $56^{\circ} \mathrm{C}$. They were dewaxed in xylene, rehydrated through a graded ethanol series, and washed with phosphate-buffered saline. Antigen retrieval was achieved by heat treatment in a pressure-cooker for $3 \mathrm{~min}$ in $10 \mathrm{mM}$ citrate buffer ( $\mathrm{pH}$ 6.5). Endogenous peroxidase was blocked. A rabbit polyclonal antibody, against aminoacids 1202 at the amino terminus of FLIP was used (H-202, Santa Cruz, Santa Cruz, CA, USA; dilution 1:10). After incubation, the reaction was visualized with the EnVision Detection Kit (DAKO, Glostrup, Denmark) using diaminobenzidine chromogen as substrate. Sections were counterstained with hematoxylin. Appropriate external and internal positive and negative controls were used. Antigen preservation was verified with vimentin and Ki-67 immunostaining.

Immunohistochemistry was evaluated by two pathologists who followed uniform pre-established criteria. Immunoreactivity was graded semiquantitatively by considering the percentage and intensity of the staining.

A histological score was obtained from each sample, which ranged from 0 (no immunoreaction) to 300 (maximum immunoreactivity). The score was obtained by applying the following formula Hscore $=1 X(\%$ light staining $)+2 X(\%$ moderate staining) $+3 X$ (\% strong staining). Since each TMA included two different tumor cylinders from each case, immunohistochemical evaluation was performed after examining both samples. The apoptotic index was calculated by the percentage of apoptotic bodies in H\&E-stained sections, after evaluating a minimal number of 1000 cells.

The reproducibility of TMA immunostaining was confirmed by comparison of TMA results with those obtained in sections from the corresponding paraffin blocks of 37 randomly selected cases. The overall concordance was $89.2 \%$. The Kappa index of agreement between the two methods ranged from 0.68 to 0.83 .

FLIP immunostaining was correlated with histological type and grade, stage, and the apoptotic index. The Fisher's exact test was used to assess association between categorical variables. The Wilcoxon rank sum and the Kruskal-Wallis tests were used to assess association between continuous and categorical variables. The Spearman rank correlation test was used to assess association between continuous and/or ordinal variables.

\section{Reagents, Cell lines, Culture Conditions and Transfection}

Recombinant TRIAL was obtained from Peprotech EC Ltd. (London, UK), 3-(4,5-dimethylthiazol-2-yl)- 
2,5- diphenyl tetrazolium bromide assay (MTT), and actinomycin D and monoclonal antibody to Tubulin were from Sigma (St Louis, MO, USA). z-IETD-fmk was from Calbiochem (La Jolla, CA, USA). Monoclonal antibody to FLIP was purchased from Alexis Corp (Lausen, Switzerland). Antibodies to active caspase-3 and caspase-8 were obtained from CellSignal (Beverly, MA). siRNA to FLIP was from SantaCruz Biotechnology, Inc. (SantaCruz, CA, USA). Peroxidase-conjugated anti-mouse and antirabbit antibodies were provided by AmershamPharmacia (Uppsala, Sweden).

The Ishikawa 3-H-12 (IK) and the KLE cell lines were obtained from the American Type Culture Collection (Manassas, VA, USA). Cells were grown in Dulbeco's modified Eagle's medium (DMEM) (Sigma, St Louis, MO, USA) suplemented with 10\% Foetal Bovine Serum (Invitrogen Inc., Carlsbad, CA, USA), $1 \mathrm{mM}$ HEPES (Sigma, St Louis, MO, USA), $1 \mathrm{mM}$ sodium pyruvate (Sigma, St Louis, MO, USA), $2 \mathrm{mM}$ L-glutamine (Sigma, St Louis, MO, USA) and $1 \%$ of penicillin/streptomicin (Sigma, St Louis, MO, USA) at $37^{\circ} \mathrm{C}$ with saturating humidity and $5 \% \mathrm{CO}_{2}$. Transfections were performed with Lipofectamine 2000 reagent (Invitrogen Inc., Carlsbad, CA, USA) following the manufacturers instructions.

\section{Western Blot Analysis}

Endometrial carcinoma cell lines were washed with cold PBS and lysed with lysis buffer ( $2 \%$ SDS, $125 \mathrm{mM}$ Tris-Hcl pH 6.8). Protein concentrations were determined with the Protein assay Kit (BioRad, Richmon, CA, USA). Equal amounts of proteins were subjected to SDS-PAGE and transferred to PVDF membranes (Millipore, Bedford, MA, USA). Nonspecific binding was blocked by incubation with TBST (20 mM Tris-Hcl pH 7.4, $150 \mathrm{mM} \mathrm{NaCl,}$ $0.1 \%$ Tween-20) plus $5 \%$ of nonfat milk. Membranes were incubated with the following antibodies: antiFLIP NF6, anti-caspase-8, anti-active-caspase-3 or anti-tubulin. Signal was detected with ECL Advance (Amersham-Pharmacia, Buckinghamshire, UK).

\section{Caspase-8 Activity}

Cells were treated with the indicated conditions for $12 \mathrm{~h}$ and harvested with lysis buffer containing $50 \mathrm{mM}$ HEPES, pH 7.4, $100 \mathrm{mM} \mathrm{NaCl}, 1 \mathrm{mM}$ EDTA, 0.1\% 3-[(3 cholamidopropyl)dimethyl-ammonio]-1propanesulfonate (CHAPS), $10 \%$ saccharose, $5 \mathrm{mM}$ dithiothreitol. Cell lysates were centrifuged at $10000 \mathrm{~g}$, and protein extracts were quantified with the Protein assay Kit (Bio-Rad, Richmon, CA, USA). Equal amounts of protein were loaded on M96 multiwell plates and the colorimetric substrate $N$ acetyl-Ile-Glu-Thr-Asp $p$-nitroanilide (Ac-IETD$p N A$ ) (Biomol, Plymouth, PA, USA) was added. Samples were incubated at $37^{\circ} \mathrm{C}$, and the absorbance at $405 \mathrm{~nm}$ was read in a microplate reader. Results were expressed as a fold induction over basal.

\section{Cell Viability Assays and Assessment of Apoptosis}

Cell viability was determined by MTT assay. Endometrial carcinoma cells were plated on M96 well plates at $15 \times 10^{3}$ cells per well. After the indicated treatments, cells were incubated for $2-3 \mathrm{~h}$ with $0.5 \mathrm{mg} / \mathrm{ml}$ of MTT reagent, lysed with DMSO. Absorbance was measured at $595 \mathrm{~nm}$ in a microplate reader (Bio-Rad, Richmon, CA, USA).

Hoechst staining was performed by adding Hoechst dye to a final concentration of $0.5 \mathrm{mg} / \mathrm{ml}$ to each M96 well. Cells were counted under epifluorescence microscope (Leica Microsystems).

\section{RNA Extraction and RT-PCR}

Total RNA was extracted using the TRIZOL reagent (Invitrogen Inc., Carlsbad, CA, USA) as indicated by the manufacturer. Of total RNA, $1 \mu \mathrm{g}$ was subjected to reverse transcription for $1 \mathrm{~h}$ at $42^{\circ} \mathrm{C}$ with the Multiscrive reverse transcription kit (Applied biosystems Inc., Foster City, CA, USA). cDNA was amplified by PCR using FLIP-specific set of primers (forward: 5'-GGACCTTGTGGTTGAGTTGG-3', reverse: $5^{\prime}$-TCTTCACTGGTTCTTGTTGAGC-3') for each gene plus the actin primers. Cycling conditions were as follows: $45 \mathrm{~s}$ at $94^{\circ} \mathrm{C}, 45 \mathrm{~s}$ at $55^{\circ} \mathrm{C}$ and $45 \mathrm{~s}$ at $72^{\circ} \mathrm{C}$ for 31 cycles. PCR products were resolved in an $8 \%$ acrylamide-bisacrylamide gel.

\section{Results}

\section{FLIP is Frequently Expressed in Endometrial Carcinoma, and Shows a Correlation with Stage}

In all, 10 cylinders were missed in either the construction of the tissue microarray, section or staining procedures. Six cases were excluded because of unreliable staining of vimentin and Ki-67. Thus, FLIP immunostaining was evaluated, in at least one cylinder, in 88 cases. Cytoplasmic staining was observed in 34 cases (43\%), and the Hscore varied from 0 to $170($ mean $=33.98)$ (Figure 1$)$. There was a statistically significant difference in FLIP expression between Stage I and II tumors $(P=0.016)$. The mean Hscore was 25.74 for stage I tumors, and 62.7 for stage II tumors. However, FLIP staining did not show statistically significant correlation with histological type $(P=0.723)$, grade $(P=0.914)$ or apoptotic index $(P=0.177)$ (Table 1$)$.

\section{Actinomycin D Downregulates FLIP and Sensitizes Endometrial Carcinoma Cells to TRAIL-Induced Apoptosis}

TRAIL is able to induce apoptosis in a wide variety of tumor cells. However, several types of tumors 
888
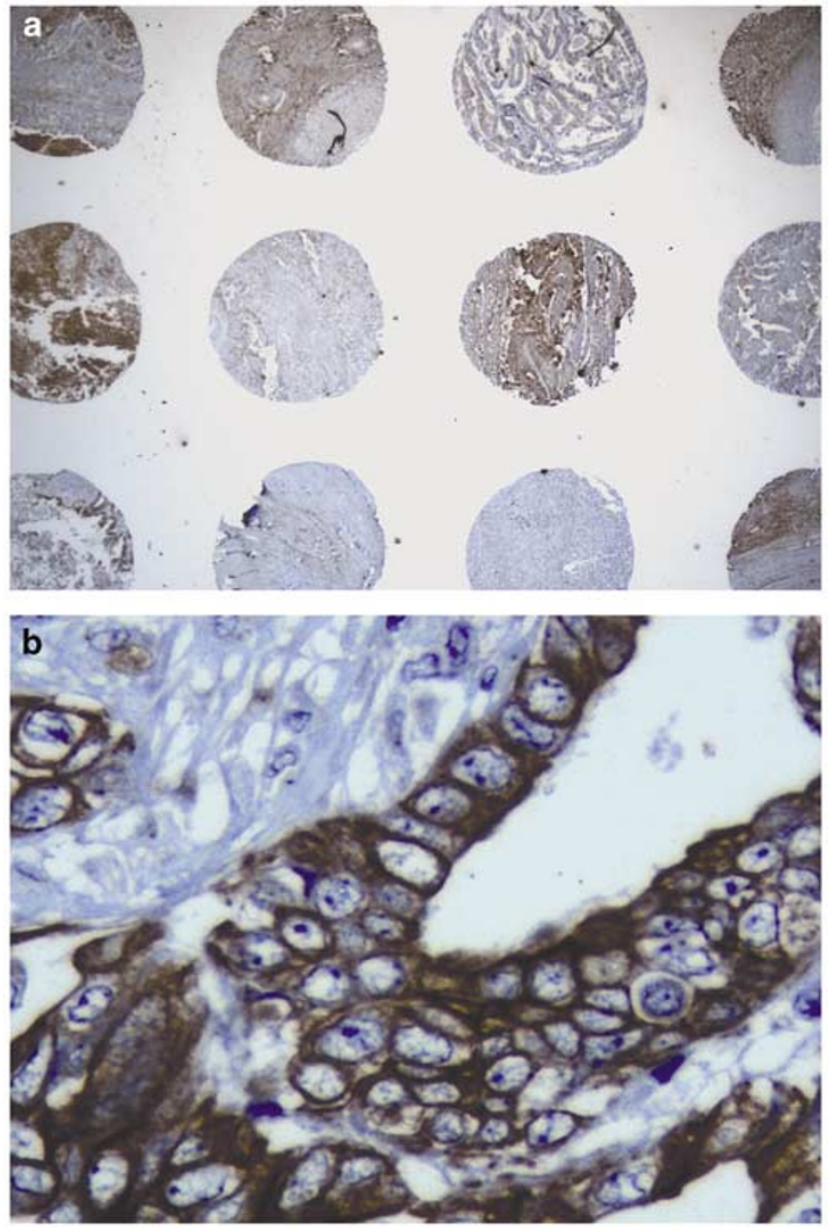

Figure 1 FLIP is frequently expressed in endometrial carcinoma. A tissue microarray constructed from 95 endometrial carcinoma was stained with an anti-FLIP antibody. A significant number of cylinders showed intense staining for FLIP (a) with a cytoplasmic pattern (b).

Table 1 Summary of the statistical analysis

\begin{tabular}{lcccc}
\hline Protein & $\begin{array}{c}\text { Histological } \\
\text { type (EEC/NEEC) }\end{array}$ & $\begin{array}{c}\text { Tumor } \\
\text { grade }\end{array}$ & $\begin{array}{c}\text { Pathological } \\
\text { stage }\end{array}$ & $\begin{array}{c}\text { Apoptotic } \\
\text { index }\end{array}$ \\
\hline Flip & $P=0.723$ & $P=0.914$ & $P=0.016$ & $P=0.177$
\end{tabular}

display TRAIL resistance. First, we explored the ability of endometrial carcinoma cell lines to undergo apoptosis after TRAIL treatment. IK and KLE cells were treated for several days with $50 \mathrm{ng} / \mathrm{ml}$ of TRAIL. Exposure to TRAIL even for long periods of time did not significantly reduce the viability of either cell line. Only a 5-10\% reduction in cell viability was observed during the first $24-72 \mathrm{~h}$ in culture (Figure 2a).

A classical approach to sensitize resistant cells to TRAIL-triggered apoptosis is the inhibition of transcription or translation. ${ }^{11,22}$ Such inhibition may block the expression of antiapoptotic mole-
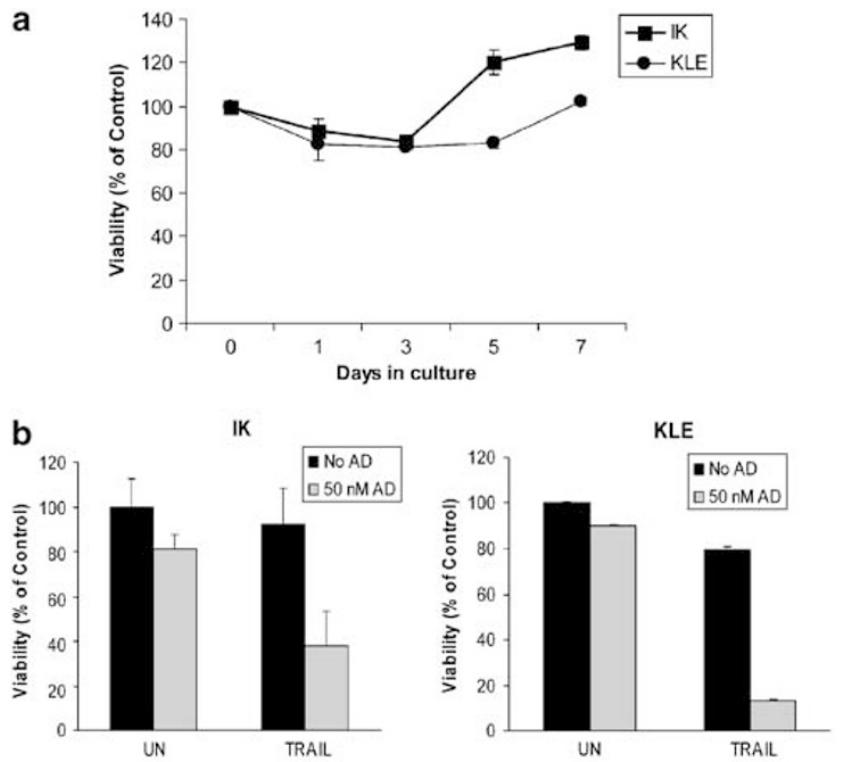

C

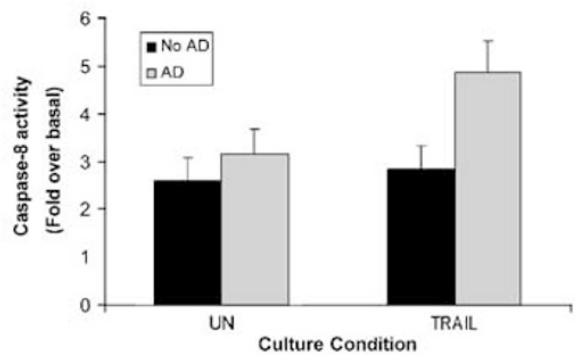

Figure $2 \mathrm{AD}$ sensitizes IK and KLE to TRAIL-induced apoptosis. IK and KLE cell lines were incubated with $50 \mathrm{ng} / \mathrm{ml}$ of TRAIL and percentage viability was assessed by MTT viability assay after 1 , 3,5 or 7 days (a). IK and KLE cells were untreated (UN) or treated with $50 \mathrm{ng} / \mathrm{ml}$ of TRAIL with or without $50 \mathrm{nM}$ actinomycin D (AD) for $24 \mathrm{~h}$ (IK) or $48 \mathrm{~h}$ (KLE). Cell viability was assessed by MTT and expressed as percentage of the control (b). IK cells were untreated (UN) or treated with $50 \mathrm{ng} / \mathrm{ml}$ of TRAIL with or without $50 \mathrm{nM}$ actinomycin D (AD) for $12 \mathrm{~h}$ and caspase-8 activity was measured (c).

cules. Cotreatment of TRAIL with actinomycin D (an inhibitor of transcription) resulted in a significant reduction of cell viability of IK and KLE cells, together with an increase of caspase-8 activity (Figure 2b, c). Actinomycin D alone caused marginal changes on either cell viability or caspase-8 activity. Altogether these results indicate that actinomycin D sensitizes endometrial carcinoma cell lines to apoptotic cell death triggered by activation of death receptors. One of the key regulators of TRAIL apoptosis is FLIP. High levels of FLIP block caspase- 8 binding to the DISC, uncoupling ligand binding from caspase activation, and ultimately inhibiting cell death. Therefore, regulation of FLIP levels may be a determinant in the response to TRAIL. Treatment of IK and KLE cells with actinomycin D plus TRAIL resulted in a dramatic downregulation of FLIP mRNA and protein (Figure 3) suggesting that high levels of FLIP may 
be involved in TRAIL resistance to apoptosis in endometrial carcinoma.

\section{Overexpression of FLIP-L Overcomes TRAIL-Induced Apoptosis}

To ascertain whether high levels of FLIP rescue endometrial carcinoma cell lines from TRAIL plus actinomycin D-induced apoptosis, we transiently transfected IK cells with a plasmid coding for FLIPL. After $24 \mathrm{~h}$ of transfection to allow FLIP-L expression, cells were treated with TRAIL or TRAIL plus actinomycin D and apoptotic nuclei were visualized by Hoechst staining. As shown in Figure 4, ectopic expression of FLIP-L resulted in an increase of viable cells in the culture and consequent reduction of nuclei displaying apoptotic morphology, suggesting that FLIP-L overcomes cell death triggered by TRAIL plus actinomycin D. These results indicate that expression of FLIP is sufficient to promote TRAIL resistance in endometrial carcinoma cells. To further investigate the involvement of caspase-8 in transducing TRAIL plus actinomycin D cell killing, we carried out a viability experiment with IK and KLE cells treated with the specific caspase-8

a

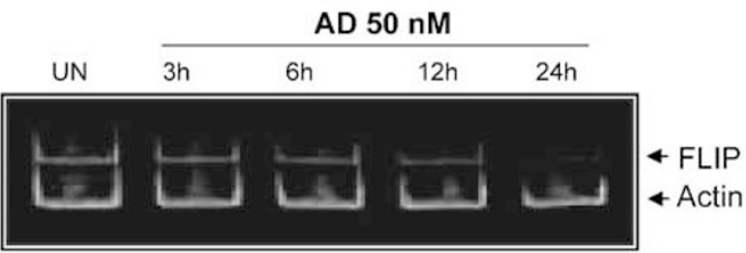

b

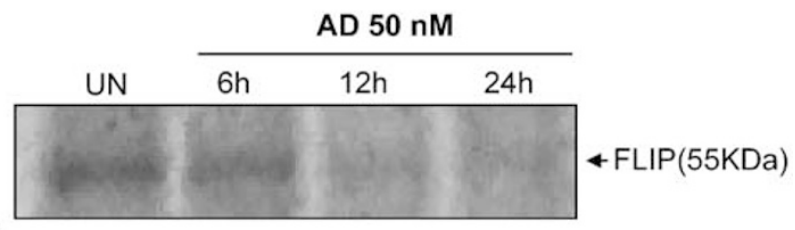

IK
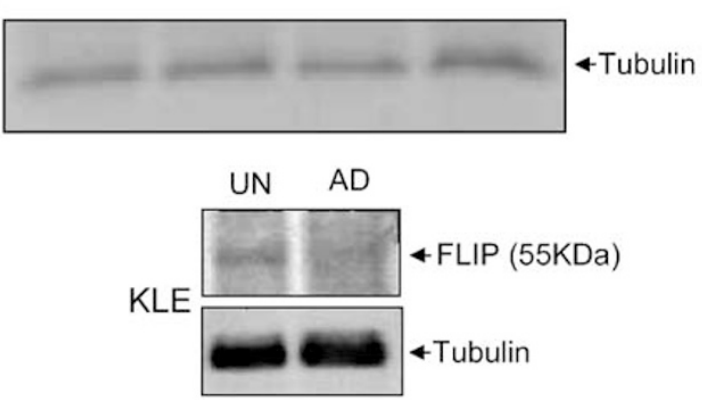

Figure $3 \mathrm{AD}$ causes downregulation of FLIP mRNA and protein. IK cells were treated for the indicated times with $50 \mathrm{nM}$ of actinomycin D and mRNA was subjected to RT-PCR using specific primers for FLIP and Actin (a). IK cells were treated with $50 \mathrm{nM}$ $\mathrm{AD}$ or left untreated (UN) for the indicated times. Cell lysates were subjected to western blot with anti-FLIP antibody (upper panel) and Tubulin antibody (lower panel). KLE cells were treated with $50 \mathrm{nM} \mathrm{AD}$ or left untreated (UN) for $24 \mathrm{~h}$ and cell lysates were analyzed by Western blot with anti-FLIP (upper panel) or antitubulin (lower panel) antibodies (b). inhibitor z-IETD-fmk. Incubation with such an inhibitor completely prevented the cell death induced by TRAIL plus actinomycin D.

a

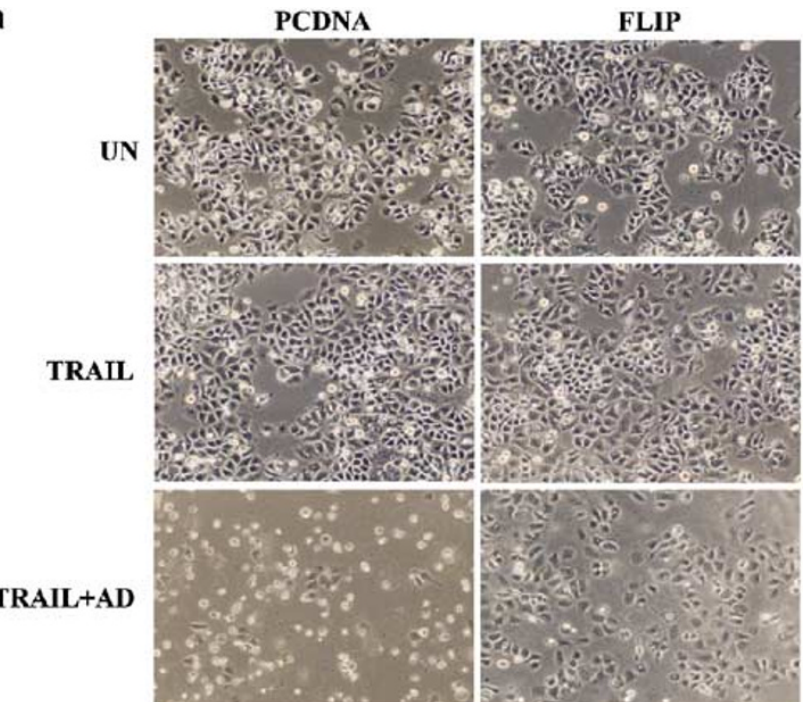

b

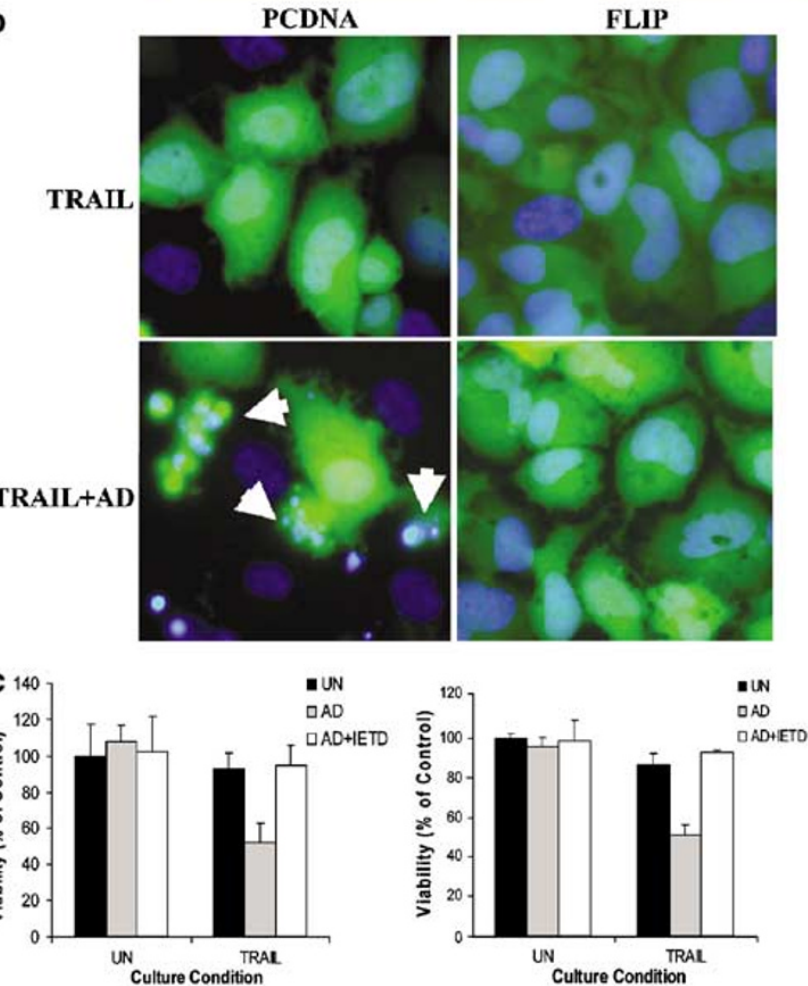

Figure 4 Caspase-8 inhibitors and overexpression of FLIP overcomes TRAIL induced apoptosis. IK were transiently cotransfected with the yellow fluorescent protein plus FLIP cDNA (FLIP) or the empty vector (PCDNA3). After $24 \mathrm{~h}$ cells were left untreated (UN), treated with $50 \mathrm{ng} / \mathrm{ml}$ of (TRAIL) or TRAIL plus actinomycin D (TRAIL + AD) and incubated for additional $24 \mathrm{~h}$. Representative fields of IK cells transfected with PCDNA3 or FLIP, and incubated for additional $24 \mathrm{~h}$ with TRAIL alone or TRAIL plus AD and visualized under contrast phase (a) or stained with Hoechst after $24 \mathrm{~h}$ of treatment (b). IK and KLE cells were incubated with Actinomycin D with or without $100 \mu \mathrm{M}$ z-IETD-fmk and left untreated or incubated with $50 \mathrm{ng} / \mathrm{ml}$ of TRAIL. Cell viability was assessed by MTT assay . 


\section{Downregulation of Endogenous FLIP Expression Restores TRAIL-Induced Apoptosis}

To confirm the role of endogenous FLIP in the prevention of TRAIL-triggered apoptosis, IK and KLE cells were transfected with FLIP siRNA and incubated for $24 \mathrm{~h}$ to allow the specific inhibition of FLIP expression without effects on a homologous protein such as caspase-8 (Figure 5a). Afterwards, we examined the siRNA-mediated downregulation of endogenous FLIP on TRAIL incubation. IK and KLE cells were transfected and, after $24 \mathrm{~h}$, exposed to TRAIL, and incubated 24 additional hours. Apoptosis was assessed by quantification of apoptotic nuclei visualized by Hoechst staining. TRAIL incubation induced a marked increase of apoptotic figures in IK and KLE cultures transfected with FLIP siRNA, when compared to the control siRNA transfected cells (Figurea 5b, c). Furthermore, IK and KLE cells transfected with FLIP siRNA displayed an increased cleavage of caspase- 8 and caspase-3 activation, indicating that the intracellular levels of FLIP inhibited caspase activation and thus, cell death (Figure 6). FLIP siRNA transfected KLE cells displayed an increase of apoptotic cell death and caspase activation even in the absence of TRAIL. These results provide clear evidence that the expression of FLIP in these cell lines is sufficient to cause resistance to TRAILinduced apoptosis.

\section{Discussion}

Apoptosis is a key process in the regulation of cellular homeostasis. Alterations of apoptosis plays an important role in development and progression of cancer. Deregulation of proteins involved in apoptosis control may result in cell populations that escape of apoptotic cell death. ${ }^{1}$ The lack of response to such stimuli can produce a survival advantage, and the expansion of a population of neoplastic cells. Moreover, defects in the apoptotic pathway can make cancer cells resistant to therapy.

Several proapoptotic members of the TNF superfamily trigger the 'extrinsic' apoptotic pathway. ${ }^{23}$ Among these members, TRAIL has raised interest as a potential anti-cancer agent, because of its ability to trigger apoptosis in cancer cells without affecting normal cells. ${ }^{2,3}$ However, an increasing number of tumor types show mechanisms of TRAIL resistance. In these tumors, cell death is blocked as a result of molecular alterations of proteins regulating extrinsic apoptotic pathway. In the present study, we demonstrated that FLIP is frequently expressed in endometrial carcinoma cells and suggested, for the first time, that FLIP may play a significant role in resistance of endometrial cancer cells to TRAIL-induced apoptosis.
The results of the present study showed that FLIP expression is frequent in endometrial carcinomas. Interestingly, FLIP expression did not correlate with histological type or grade, suggesting that it may be involved in the process of apoptosisresistance of both type I (endometrioid) and type II (nonendometrioid) endometrial carcinomas. These two types of endometrial carcinomas do not show only different morphological features, but exhibit significant clinical and molecular differences. ${ }^{24}$

The possible effects of FLIP and TRAIL in the development of apoptotic cell death in endometrial carcinoma cells have not been addressed so far. We first assessed the viability of two endometrial carcinoma cell lines after exposition to TRAIL. Treatment of both IK and KLE with TRAIL failed to cause a significant reduction on viability. Only a marginal reduction of $5-10 \%$ was observed on the first $24-72 \mathrm{~h}$ in culture. However, incubation of these cell lines with actinomycin D (an inhibitor of transcription) plus TRAIL resulted in a marked decrease of cell viability caused by apoptotic death. Treatment with inhibitors of transcription or translation, such as actinomycin D or cycloheximide, has been shown to be effective in sensitization of different tumor cells to members of the TNF superfamily. These inhibitors may cause the reduction of the expression of some intracellular proteins that inhibit different steps of the apoptotic cascade. The results obtained with actinomycin $\mathrm{D}$ treatment indicate that these endometrial carcinoma cell lines may express molecules that are able to block TRAIL-induced apoptosis. Consistently, the cell death caused by actinomycin treatment is accompanied by an increase of caspase-8. These results enabled us to study intracellular proteins involved in the regulation of apoptotic signalling by TRAIL. One of such regulators is FLIP, which competes with caspase-8 for the binding to FADD. Therefore, the expression of FLIP could be important as a mechanism to suppress apoptosis.

An RT-PCR and Western blot analysis on a time course exposition to actinomycin $\mathrm{D}$ showed a marked decrease in the levels of both FLIP mRNA and protein suggesting that FLIP could be one of the mechanisms of this resistance. These results are concordant with previous studies that found decreased levels of FLIP after treatment with inhibitors of transcription. ${ }^{25,26}$ To investigate whether the decreased FLIP expression was responsible for TRAIL resistance, we transfected endometrial carcinoma cells with a plasmid coding for FLIP. The transfection assays resulted in a prevention of TRAIL-induced apoptosis, which indicated that actinomycin $\mathrm{D}$ regulates the activation of the extrinsic apoptotic pathway. Furthermore, the incubation of the endometrial carcinoma cells with an inhibitor of caspase-8 (z-IETD-fmk) resulted in a complete inhibition of apoptosis. Altogether, these 


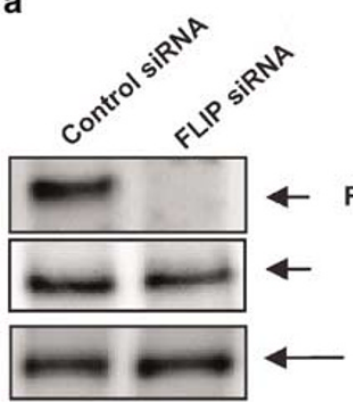

Ishikawa

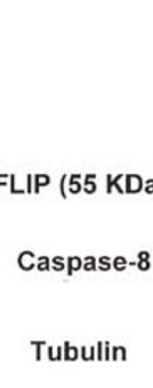

ulin

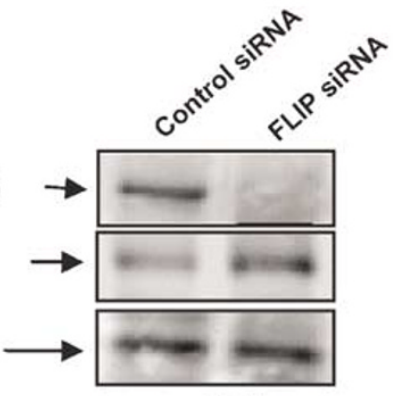

KLE b
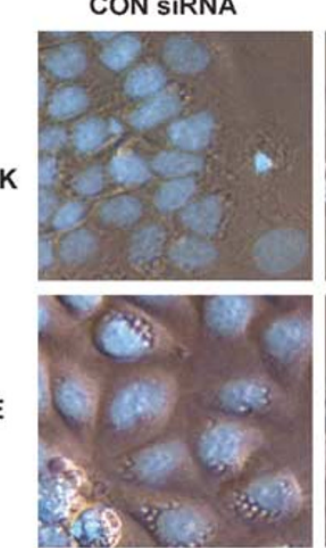

KLE
C
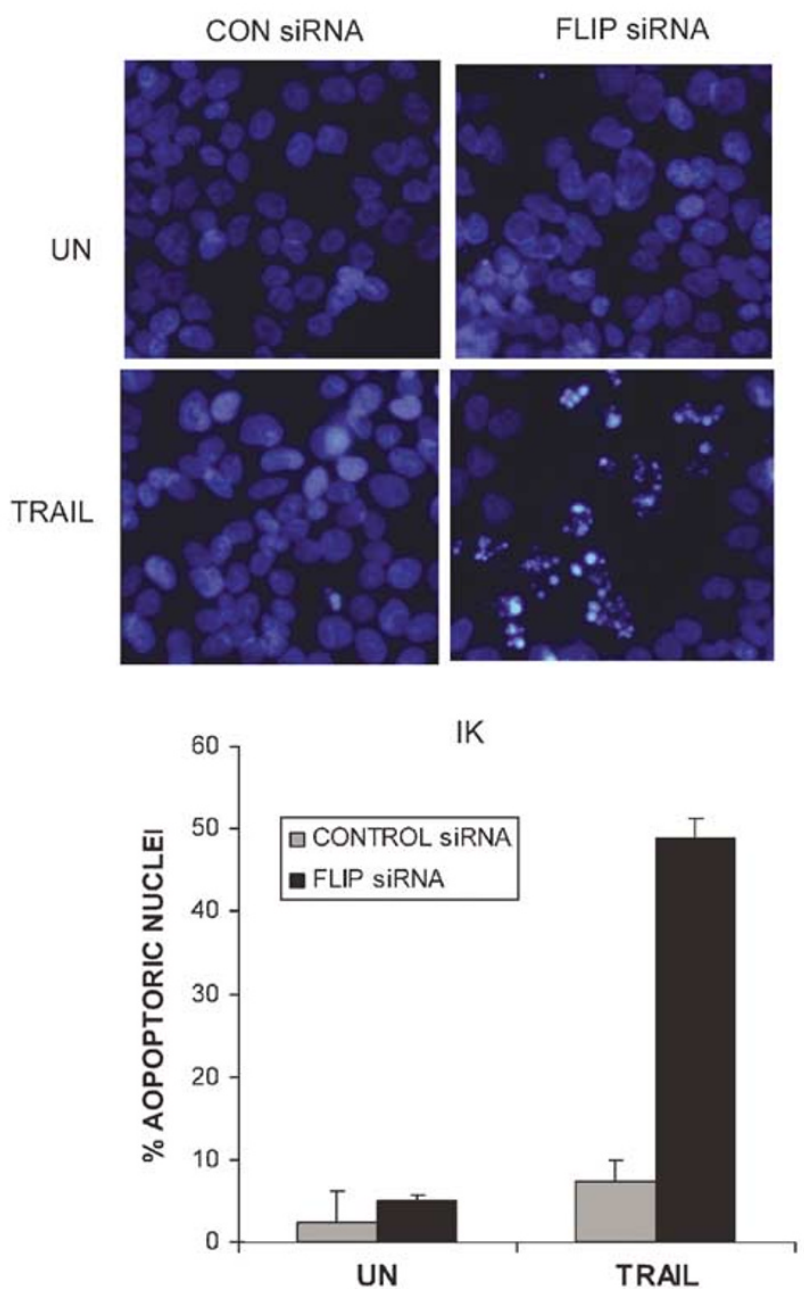
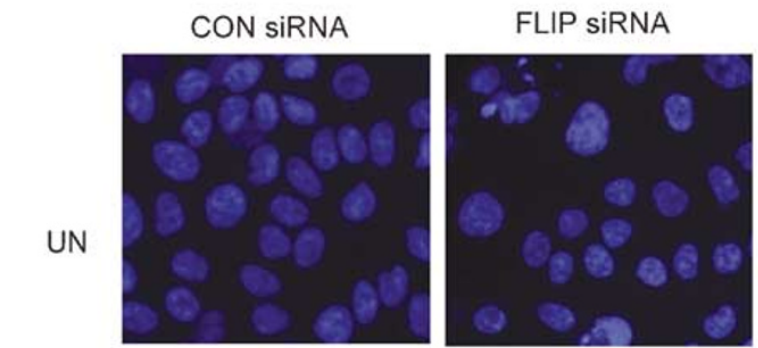

TRAIL
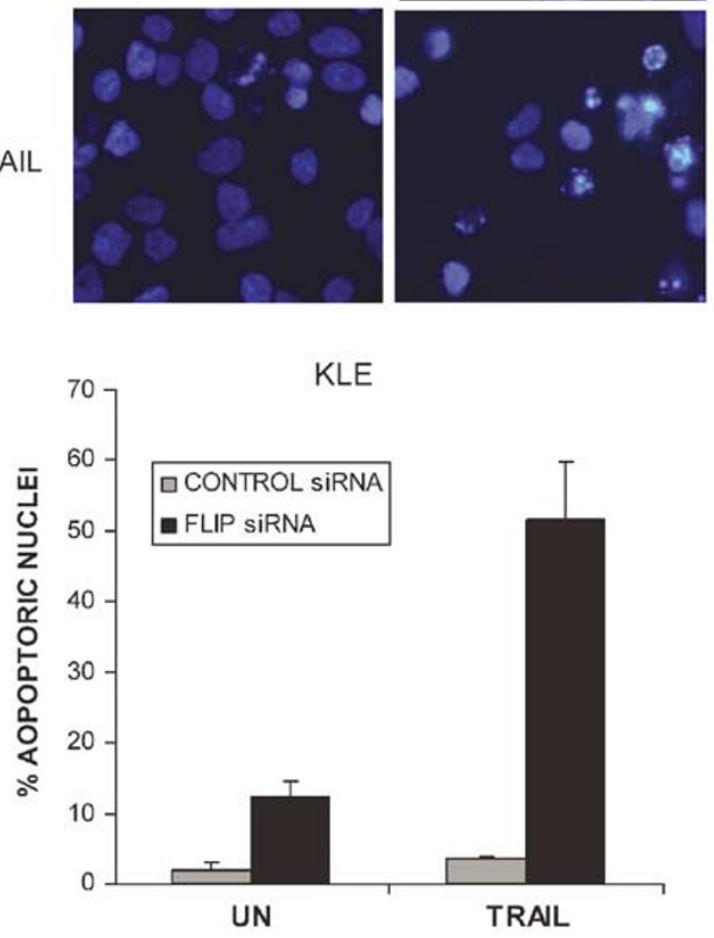

Figure 5 Downregulation of FLIP by specific siRNA sensitizes IK and KLE cells to TRAIL-induced apoptosis. IK and KLE cells were transfected with either FLIP siRNA or control siRNA. After $24 \mathrm{~h}$ protein cell lysates were subjected to Western blot with antibodies against caspase-8, FLIP or tubulin as a control of specificity and protein loading. FLIP siRNA reduces the expression of endogenous FLIP (a). Micrographs showing Hoechst staining and Hoffman optical image of FLIP siRNA transfected IK and KLE after $24 \mathrm{~h}$ of treatment with $50 \mathrm{ng} / \mathrm{ml}$ of TRAIL (b). IK and KLE cells were transfected with either control or FLIP siRNA and treated or not with $50 \mathrm{ng} / \mathrm{ml}$ of TRAIL. Micrographs show representative fields IK and KLE cells stained with Hoechst dye to evidence the apoptotic nuclear morphology. The graphs represent a quantification of nuclei displaying nuclear apoptotic morphology in IK and KLE cells (c).

data provide evidence that FLIP is, at least, one of the proteins that are involved in apoptosis resistance to TRAIL.
Direct evidence of the role of endogenous FLIP in TRAIL apoptosis resistance on endometrial carcinoma cells is provided by treatment with specific 
892

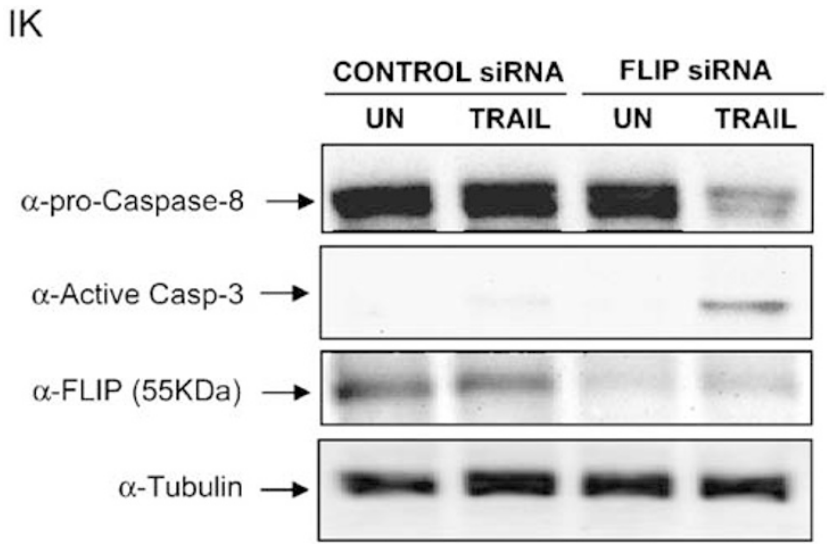

\section{KLE}
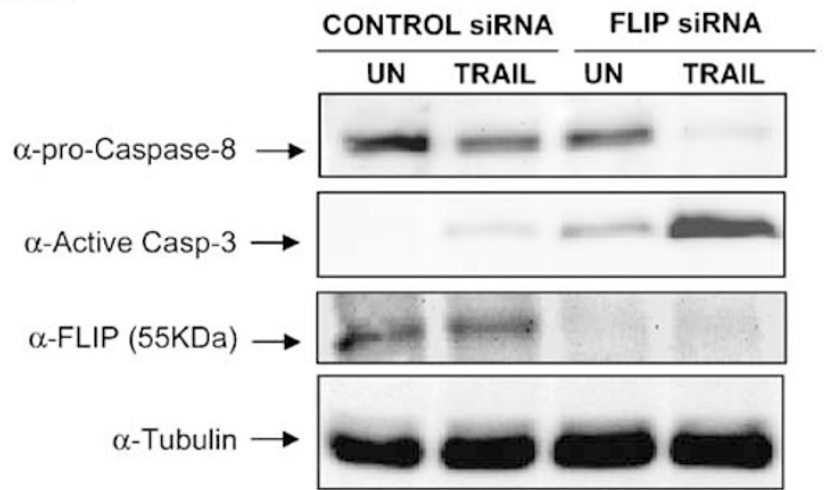

Figure 6 Downregulation of FLIP by specific siRNA activates caspase-8 and caspase-3. IK and KLE cells transfected with either control siRNA or FLIP siRNA, after $24 \mathrm{~h}$ cultures were treated with $50 \mathrm{ng} / \mathrm{ml}$ of TRAIL and incubated for further $24 \mathrm{~h}$. Protein cell lysates were subjected to Western blot with antibodies agains active caspase-3, procaspase-8, FLIP or tubulin. FLIP siRNA transfected cells show an increase of active caspase- 3 and decrease of procaspase-8 levels.

siRNA targeting FLIP. Transfection of both IK and KLE cells with FLIP siRNA resulted in a marked decrease in cell viability after TRAIL exposition. This was accompanied by processing of both caspase- 8 and caspase- 3 suggesting activation of the extrinsic pathway. The fact that endogenous FLIP inhibition induced apoptosis indicates that FLIP is necessary and sufficient to cause resistance to TRAIL-induced apoptosis. KLE cells showed an increase of apoptotic nuclei as well as caspase-3 and caspase-8 processing after $24 \mathrm{~h}$ of transfection with FLIP siRNA, but without exogenous supplied TRAIL to the medium. This result suggests that KLE cells may produce an endogenous factor that may promote self-induced cell death in an autocrine manner.

Although several studies have demonstrated an anti-apoptotic role for FLIP by interfering caspase-8 recruitment to the DISC, some recent evidences suggest that the amount of FLIP that is present in the DISC may be responsible for either suppressing or inducing apoptosis. ${ }^{27}$ Thus, FLIP may cause activation of both caspase- 8 and caspase-10. ${ }^{28,29}$ Our findings demonstrated that in endometrial carcinoma cell lines, the endogenous levels of expression of FLIP may inhibit caspase activation. The results also showed that downregulation of FLIP enables caspase cleavage and apoptosis.

There is evidence suggesting a possible role of FLIP in the resistance to apoptosis in cancer. FLIP is a human cellular homologue of viral FLICE-inhibitory proteins, which are expressed by $\gamma$-herpes viruses, and were shown to be capable of blocking CD95-mediated apoptosis through association with the receptor in the DISC. ${ }^{16}$ In cells that are latently infected with human herpes virus 8 , v-FLIP is expressed at low levels but its expression is increased in Kaposi sarcomas. Human melanomas and a murine B-cell lymphoma cell line have been shown to express high levels of FLIP, which interferes with apoptosis induction by death receptors. On the other hand, there is high constitutive expression of FLIP in Reed-Sternberg cells of Hodgkin's lymphomas; and some evidences suggest that it prevents FAS-induced apoptosis in these cells. ${ }^{30,18}$ There are also evidences that FLIP may inhibit the extrinsic apoptotic pathway in carcinomas of the prostate, ${ }^{17}$ the stomach, ${ }^{19}$ and the urinary bladder, ${ }^{20}$ and melanoma. ${ }^{31}$ On the other hand, FLIP has also been shown to be expressed in ovarian cancer, ${ }^{32}$ a gynecologic type of tumor very similar to endometrial carcinoma. Interestingly, in some types of tumor, such as in carcinomas of the urinary bladder, FLIP expression was shown to correlate with advanced stage. ${ }^{20}$ Similarly, in the present series, FLIP expression also showed a statistically significant difference between stage I and II endometrial carcinomas, which may suggest that resistance to TRAIL-mediated apoptosis could be related to tumor progression.

Finally, it is worth mentioning that FLIP is an important target of the nuclear factor-Kappa B (NF$\kappa \mathrm{B})$ signalling pathway. ${ }^{33} \mathrm{NF}-\kappa \mathrm{B}$ encompasses different members of a family of transcription factors, involved in the regulation of genes encoding cytokines, cytokine receptors, and cell adhesion molecules, that drive immune and inflammatory responses. However, NF- $\kappa \mathrm{B}$ has been recently found related to carcinogenesis, by regulating genes involved in apoptosis, the cell cycle, differentiation, and cell migration. In a previous study, we demonstrated that NF- $\kappa \mathrm{B}$ family members are frequently expressed in endometrial carcinomas. ${ }^{34}$ Although assessment of FLIP expression was not the main goal of that study, we tried to correlate FLIP and NF- $\kappa$ B expression, but we did not find any statistical association between them. However, it is important to emphasize that increased expression of FLIP may result from activation of some signalling pathways, other than $\mathrm{NF}-\kappa \mathrm{B}$.

In summary, we provide evidence that FLIP is expressed in endometrial carcinoma, and that it may 
be responsible for resistance to TRAIL-induced apoptosis in this type of tumor.

\section{Acknowledgements}

This work was supported by Grants FISS 01/1656, FISS PI020227, SAF 2004-05250 and 2002XT00115. XD holds a postdoctoral fellowship supported by Department d'Universitats, Recerca i Societat de la Informació, Generalitat de Catalunya (2003RED0018). JP holds a research fellowship from the Fondo de Investigaciones Sanitarias, Ministerio de Sanidad y Consumo (BEFI-02/9007). The work was also supported by FIS (PI020051 and Redes de Investigación Cooperativa), Fundació La Caixa 2002 and Suport als Grups de Recerca (Generalitat de Catalunya).

\section{References}

1 Kaufmann SH, Vaux DL. Alterations in the apoptotic machinery and their potential role in anticancer drug resistance. Oncogene 2003;22:7414-7430.

2 Ashkenazi A, Pai RC, Fong S, et al. Safety and antitumor activity of recombinant soluble Apo2 ligand. J Clin Invest 1999;104:155-162.

3 Walczak H, Miller RE, Ariail K, et al. Tumoricidal activity of tumor necrosis factor-related apoptosisinducing ligand in vivo. Nat Med 1999;5:157-163.

4 Srivastava RK. TRAIL/Apo-2L: mechanisms and clinical applications in cancer. Neoplasia 2001;3: $535-546$.

5 Pan G, O'Rourke K, Chinnaiyan AM, et al. The receptor for the cytotoxic ligand TRAIL. Science 1997;276: 111-113.

6 MacFarlane M, Ahmad M, Srinivasula SM, et al. Identification and molecular cloning of two novel receptors for the cytotoxic ligand TRAIL. J Biol Chem 1997;272:25417-25420.

7 Walczak H, Degli-Esposti MA, Johnson RS, et al. TRAIL-R2: a novel apoptosis-mediating receptor for TRAIL. EMBO J 1997;16:5386-5397.

8 Sheridan JP, Marsters SA, Pitti RM, et al. Control of TRAIL-induced apoptosis by a family of signaling and decoy receptors. Science 1997;277:818-821.

9 Pan G, Ni J, Wei YF, et al. An antagonist decoy receptor and a death domain-containing receptor for TRAIL. Science 1997;277:815-818.

10 Pan G, Ni J, Yu G, et al. TRUNDD, a new member of the TRAIL receptor family that antagonizes TRAIL signalling. FEBS Lett 1998;424:41-45.

11 LeBlanc HN, Ashkenazi A. Apo2L/TRAIL and its death and decoy receptors. Cell Death Differ 2003; 10:66-75.

12 Bodmer JL, Holler N, Reynard S, et al. TRAIL receptor2 signals apoptosis through FADD and caspase-8. Nat Cell Biol 2000;2:241-243.

13 Sprick MR, Weigand MA, Rieser E, et al. FADD/ MORT1 and caspase-8 are recruited to TRAIL receptors 1 and 2 and are essential for apoptosis mediated by TRAIL receptor 2. Immunity 2000;12:599-609.
14 Kischkel FC, Lawrence DA, Chuntharapai A, et al. Apo2L/TRAIL-dependent recruitment of endogenous FADD and caspase-8 to death receptors 4 and 5 . Immunity 2000;12:611-620.

15 Thome M, Schneider P, Hofmann K, et al. Viral FLICEinhibitory proteins (FLIPs) prevent apoptosis induced by death receptors. Nature 1997;386:517-521.

16 Irmler $\mathrm{M}$, Thome $\mathrm{M}$, Hahne $\mathrm{M}$, et al. Inhibition of death receptor signals by cellular FLIP. Nature 1997; 388:190-195.

17 Zhang X, Jin TG, Yang H, et al. Persistent c-FLIP(L) expression is necessary and sufficient to maintain resistance to tumor necrosis factor-related apoptosisinducing ligand-mediated apoptosis in prostate cancer. Cancer Res 2004;64:7086-7091.

18 Dutton A, O'Neil JD, Milner AE, et al. Expression of the cellular FLICE-inhibitory protein (c-FLIP) protects Hodgkin's lymphoma cells from autonomous Fasmediated death. Proc Natl Acad Sci USA 2004;101: 6611-6616.

19 Lee SH, Kim HS, Kim SY, et al. Increased expression of FLIP, an inhibitor of Fas-mediated apoptosis, in stomach cancer. APMIS 2003;111:309-314.

20 Korkolopoulou P, Goudopoulou A, Voutsinas G, et al. c-FLIP expression in bladder urothelial carcinomas: its role in resistance to Fas-mediated apoptosis and clinicopathologic correlations. Urology 2004;63: 1198-1204.

21 Rippo MR, Moretti S, Vescovi S, et al. FLIP overexpression inhibits death receptor-induced apoptosis in malignant mesothelial cells. Oncogene 2004;23: 7753-7760.

22 Olsson A, Diaz T, Aguilar-Santelises M, et al. Sensitization to TRAIL-induced apoptosis and modulation of FLICE-inhibitoryprotein in B chronic lymphocytic leukemia by actinomycin D. Leukemia 2001;15: 1868-1877.

23 Muppidi JR, Tschopp J, Siegel RM. Life and death decisions: secondary complexes and lipid rafts in TNF receptor family signal transduction. Immunity 2004; 21:461-465.

24 Matias-Guiu X, Catasus L, Bussaglia E, et al. Molecular pathology of endometrial hyperplasia and carcinoma. Hum Pathol 2001;32:569-577.

25 Griffith TS, Chin WA, Jackson GC, et al. Intracellular regulation of TRAIL-induced apoptosis in human melanoma cells. J Immunol 1998;161:28332840.

26 Okano H, Shiraki K, Inoue H, et al. Cellular FLICE/ caspase-8-inhibitory protein as a principal regulator of cell death and survival in human hepatocellular carcinoma. Lab Invest 2003;83:1033-1043.

27 Chang DW, Xing Z, Pan Y, et al. C-FLIP(L) is a dual function regulator for caspase-8 activation and CD95-mediated apoptosis. EMBO J 2002;21: 3704-3714.

28 Micheau O, Thome M, Schneider P, et al. The long form of FLIP is an activator of caspase- 8 at the Fas death-inducing signaling complex. J Biol Chem 2002; 277:45162-45171.

29 Boatright KM, Deis C, Denault JB, et al. Activation of caspases-8 and -10 by FLIP(L). Biochem J 2004;382: 651-657.

30 Thomas RK, Kallenborn A, Wickenhauser C, et al. Constitutive expression of c-FLIP in Hodgkin and Reed-Sternberg cells. Am J Pathol 2002;160:15211528. 
31 Zeise E, Weichenthal M, Schwarz T, et al. Resistance of human melanoma cells against the death ligand TRAIL is reversed by ultraviolet-B radiation via downregulation of FLIP. J Invest Dermatol 2004;123:746-754.

32 Abedini MR, Qiu Q, Yan X, et al. Possible role of FLICElike inhibitory protein (FLIP) in chemoresistant ovarian cancer cells in vitro. Oncogene 2004;23:6997-7004.
33 Micheau O, Lens S, Gaide O, et al. NF-kappaB signals induce the expression of c-FLIP. Mol Cell Biol 2001;21:5299-5305.

34 Pallares J, Martinez-Guitarte JL, Dolcet X, et al. Abnormalities in the NF-kappaB family and related proteins in endometrial carcinoma. J Pathol 2004;204: 569-577. 